\title{
Pregnancy with Gastric Stromal Tumor
}

\author{
Iliass Charif ${ }^{*}$, Najat Khalil1, Abdelmalek Ousadden², El Bachir Benjelloun², \\ Ouafae Slimani ${ }^{3}$, Fatima Zahra Fdili Alaoui ${ }^{3}$, Fatima Zahra Hijri, \\ Omar Mesbahi' ${ }^{4}$, Adil Ibrahimi'1, Ihsane Mellouki1

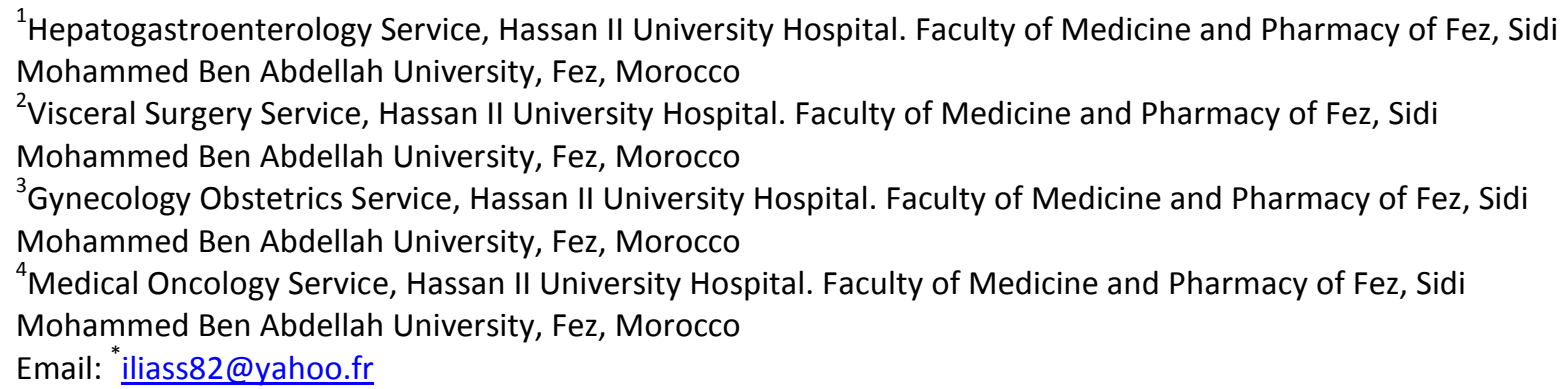

Received 15 September 2014; revised 10 October 2014; accepted 3 November 2014

Copyright (C) 2014 by authors and Scientific Research Publishing Inc.

This work is licensed under the Creative Commons Attribution International License (CC BY). http://creativecommons.org/licenses/by/4.0/

(c) (i) Open Access

\section{Abstract}

Background: Gastrointestinal stromal tumors (GIST) are the most common mesenchymal tumors of the gastrointestinal tract. GIST are rare during pregnancy. We report here the case of a patient who was admitted in our university hospital for GIST discovered during the second trimester of pregnancy. She was 42 years old, in the fifth months of pregnancy. She was admitted for biliary colic pain with vomiting. On abdominal examination, we objectified a distended abdomen with uterine height of $18 \mathrm{~cm}$ and epigastric mass of $10 \mathrm{~cm}$. Abdominal ultrasound and magnetic resonance imaging (MRI) showed a hepatic, tissue and cystic mass, developing at the expense of the left liver. Endoscopy objectified an aspect of extrinsic compression at the front of the stomach. A cesarean was scheduled at the $35^{\text {th }}$ month of pregnancy for fetal extraction and rescue. Exploration of the peritoneal cavity during surgery has objectified a hepatic highly vascularised mass reaching up to the umbilicus. This mass was unresectable. A postoperative CT scan revealed a large bilobed epigastric mass adhering to the stomach and the left liver. Histological and immunohistochemical study of hepatic process showed a gastrointestinal stromal tumor of high risk of malignancy. Conclusion: few cases have been reported in the literature on GIST during pregnancy showing the rarity of the condition that requires multidisciplinary care.

\section{Keywords}

GIST, Pregnancy, Imatinib

\footnotetext{
${ }^{*}$ Corresponding author.
}

How to cite this paper: Charif, I., Khalil, N., Ousadden, A., Benjelloun, E.B., Slimani, O., Alaoui, F.Z.F., Hijri, F.Z., Mesbahi, O., Ibrahimi, A. and Mellouki, I. (2014) Pregnancy with Gastric Stromal Tumor. Case Reports in Clinical Medicine, 3, 571-576. http://dx.doi.org/10.4236/crcm.2014.311124 


\section{Introduction}

GISTs are mesenchymal tumors that develop in the majority of cases at the expense of the stomach and small intestine, more rarely in the rectum, colon, esophagus or mesentery. They develop from Cajal cells or their precursor, and have typically CD117/KIT+ (95\%), CD34+ (70\%) phenotype. They are often associated with mutations activating genes encoding for KIT receptor (tyrosine kinase) or PDGFRA. The diagnosis of GIST during pregnancy is very rare. There are less than 20 cases reported in literature about gastrointestinal stromal tumors diagnosed during pregnancy [1]. We report the case of a gastric GIST diagnosed during pregnancy.

\section{Case}

She is a 42-years-old patient, mother of 7 children, in the fifth month of pregnancy. Admitted for treatment of moderate hepatic colic pain, dating back to seven months, atypical epigastric pain, epigastric fullness relieved by post prandial vomiting, associated with fever and general state. Clinical examination found a patient in a good general state, a distended abdomen with uterine height of $18 \mathrm{~cm}$, an epigastric smmoth and painful mass of $10 \mathrm{~cm}$. Laboratory tests showed a hypochromic microcytic anemia with a biological inflammatory syndrome. The morphological assessment including abdominal ultrasound has objectified a hepatic cystic and tissue mass, with thickened and irregular contours. Hepatic MRI found a large lesion process in the epigastric region, this lesion is heterogeneous with dual component: a liquefied center (rich in mucin and blood) and very irregular peripheral component with irregular and asymmetric internal contours. This mass is localized in the epigastric region, tacking the left liver and coming into contact with the stomach wall (small curvature) (Figure 1, Figure 2).

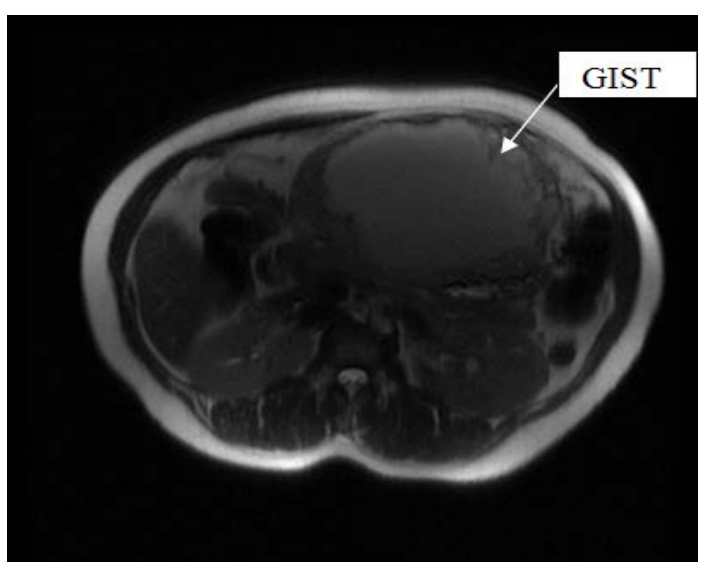

Figure 1. Axial view of the stromal tumor in MRI.

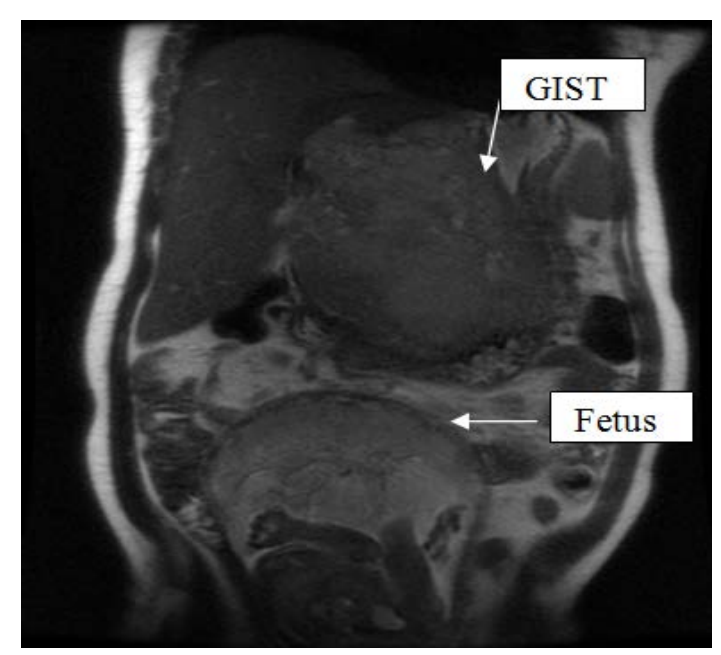

Figure 2. Sagittal view of the stromal tumor in MRI. 
A pelvic ultrasound has shown an evolutionary single-fetus pregnancy, estimated at 35 weeks of pregnancy. An upper gastrointestinal endoscopy was performed given the gastric compressive aspect of the tumor. It highlighted an aspect of extrinsic compression at the front of the stomach, without mucosa lesions.

After discussing the issue in a multidisciplinary meeting in the presence of Obstetricians, the diagnosis of gastric GIST was suspected, and the decision was to continue the pregnancy until fetal maturation and to perform more explorations. Corticosteroids have been started and monitored every week until 35 weeks. A cesarean section was performed. It allowed the extraction of a newborn of $2.5 \mathrm{~kg}$ without anomalies. The exploration of the abdomen during surgery objectified a highly vascularized mass at the expense of the liver, reaching up to the umbilicus, not resectable.

Post operative abdominal CT had objectified a large epigastric mass measuring $16 \times 21 \times 23 \mathrm{~cm}$ in diameter, limited and bilobed with dual components: fleshy peripheral component enhanced after contrast and liquefied central component with significant vasculature. This tumor mass increased comparing to the results of the MRI (Figure 3).

A liver biopsy of the mass showed a gastrointestinal stromal tumor with high risk of malignancy. Immunohistochemistry has revealed a positive CD 117 and CD 34, PS100 and AML (Figure 4).

Imatinib at dose of $400 \mathrm{mg}$ daily was started for reducing the tumor size before surgical resection.

Three months later, the size of the epigastric mass decreased but metatstasis liver appeared.

The patient underwent surgery (Figure 5) a month later, which revealed a cystic tumor of $30 \mathrm{~cm}$ in diameter, displacing the stomach back and left with an intimate contact with the underside of the left lobe of the liver. A total gastrectomy was performed, with hepatic resection of the left lobe of the liver, allowing removing quite the

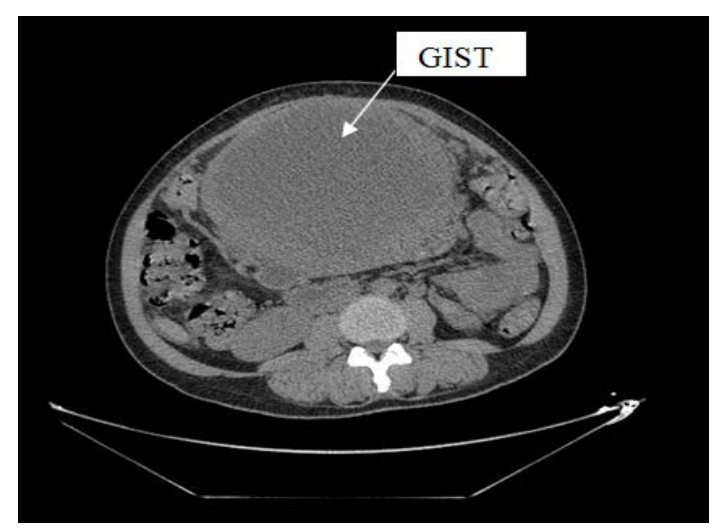

Figure 3. Axial view of the stromal tumor in abdominal CT.

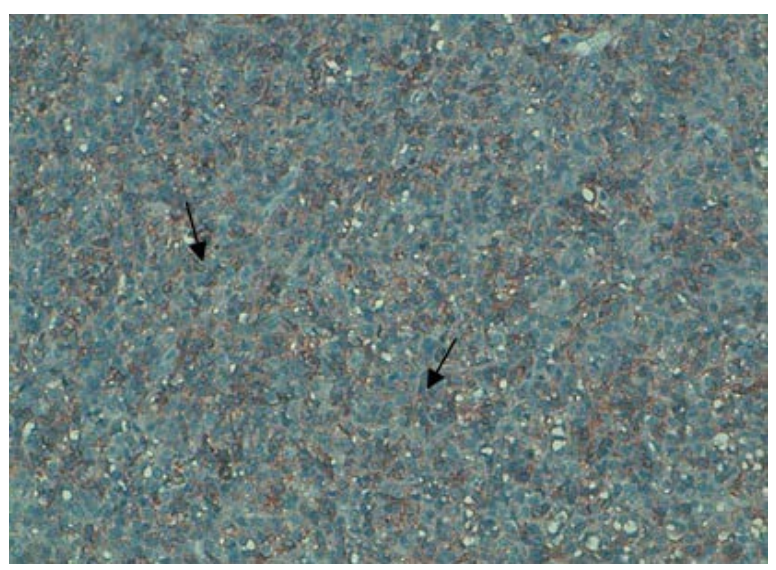

(a)

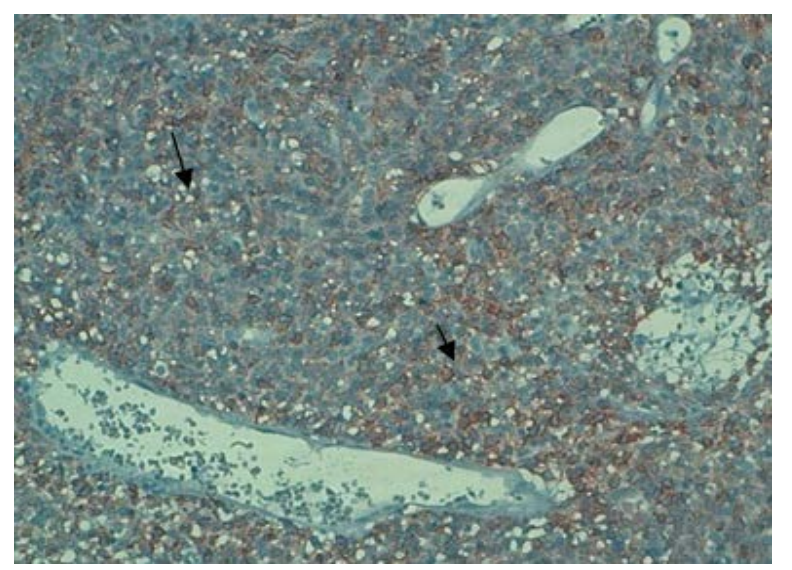

(b)

Figure 4. Immunohistochemistry. (a) positive immunohistochemistry for the CD117; (b) positive immunohistochemistry for the CD34. 


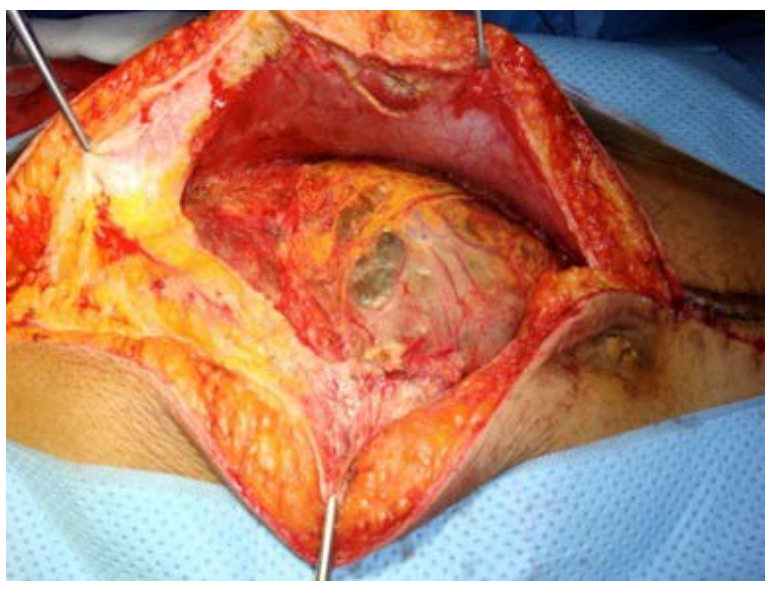

(a)

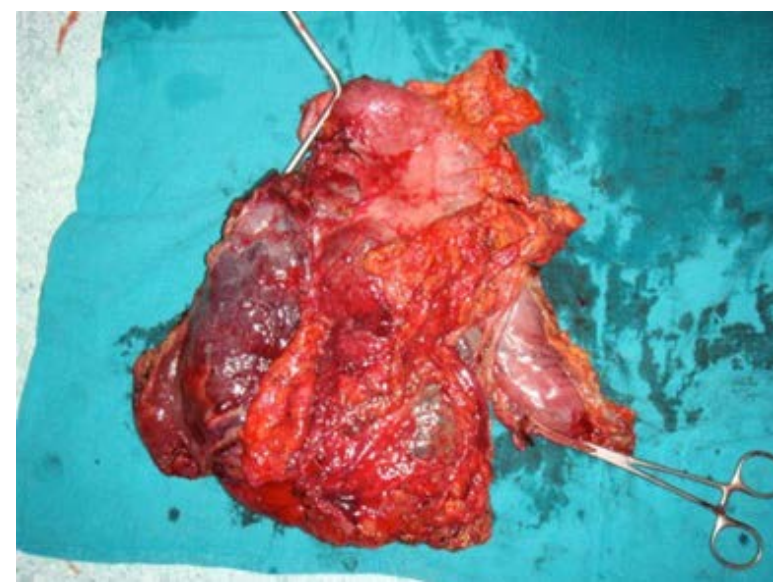

(b)

Figure 5. Macroscopic aspect of the stromal tumor. (a) at surgical exploration; (b) after resection of the stromal tumor.

tumor. Esophagus jejunum anastomosis and feeding jejunostomy were performed. The histological study showed a high grade gastric stromal tumor with necrosis of more than $50 \%$ of the tumor (due to the response to imatinib). Distal and proximal resction's limits of stomach and liver were healthy. Lymphadenectomy found 26 non-metastatic lymph nodes. A neoadjuvant treatment with imatinib $400 \mathrm{mg}$ per day was started, with good adherence, good tolerability, and a partial clinical and radiological response. However, the patient presented a neutropenia $\left(800\right.$ elements $/ \mathrm{mm}^{3}$ ), which pushed us to reduce the daily dose of imatinib to half, with good tolerance and correction of neutropenia.

\section{Discussion}

Gastrointestinal stromal tumors (GIST) are rare tumors. the incidence is aproximately 10 to 20 cases per million people and year [1]. Early diagnosis is important for preserving the prognosis. They are much rarer during pregnancy, few cases have been reported in the literature [2]. GISTs are indeed the most common mesenchymal tumors of the gastrointestinal tract. Microscopy and immunohistochemistry allowed classifying these tumors according to their phenotypes and prognosis. The two main markers identified were CD34, and KIT protein or CD 117.

The incidence of these tumors in the United States is estimated at 3000 - 4000 cases per year, with a median age of 60 years [3]. The majority of GISTs are localized in the stomach (60\%) and small intestine (30\%), especially when associated with pregnancy [1], the remaining $10 \%$ are located in the esophagus and rectum [4]. Their occurrence is sporadic in most cases, but there are some familial predisposition, such as neurofibromatosis type I and exceptional familial forms described by Carney and Stratakis or related to a constitutional mutation of KIT or PDGFRA [5].

The association of GIST to pregnancy, although rare, is not anecdotal. The challenge of this situation is to make the diagnosis early and start the efficient treatment without a major impact on the fetus. The diagnosis can be difficult because the age of onset of GIST in a pregnant woman does not fit with the peak incidence reported in the literature.

About 20\% of cases are diagnosed when performing an endoscopy for another indication. In $15 \%$ - 25\% of cases, GIST are discovered at the metastatic stage. The clinical presentation during pregnancy is not specific: gastrointestinal bleeding, unexplained anemia or abdominal mass [6]. Cases of GIST reported in the literature, show a non-specific symptoms. However, only the histological analysis allows confirming the diagnosis.

GISTs are usually well-circumscribed without encapsulation, they grow preferentially on the serosal side of the bowel wall. Macroscopically, the measurement of the maximum diameter of the primary tumor is an important parameter for evaluating the evolutionary potential [7]. It is important to sample the tumor, for the differential diagnosis with other sarcomas (e.g. liposarcoma), and because there may be variations in the proliferation index. Histoloically, cell density is generally high and homogeneous, and necrotic alterations, edema and/or bleeding are as most frequent as tumors are large. The cells are fusiform in $70 \%$ of cases, most often with a fas- 
cicular architecture, suggesting a smooth muscle proliferation. Several other tumors may express c-KIT [8] in the majority (seminoma, adenoid cystic carcinoma) or in a variable proportion of cases (melanoma, lung carcinoma, etc...). Approximately 5\% of GISTs are negative for c-KIT and then the diagnosis requires the identification of mutations of KIT or PDGFRA genes in tumor DNA [6]. The average diameter of symptomatic tumors is $6 \mathrm{~cm}$ against $1.5 \mathrm{~cm}$ for asymptomatic tumors [9]. Our patient had a tumor exceeding $20 \mathrm{~cm}$. The cases reported in the literature had tumors diameter ranging between 4 and $17 \mathrm{~cm}$ [10]. Useful tests for the diagnosis of GIST depend on the size and location of the tumor. For tumors less than $5 \mathrm{~cm}$ and gastric or colorectal localization, the diagnosis is made by endoscopy and confirmed by ultrasonography. For small GIST of the small intestine, the diagnosis is made by enteroclysis and/or enteroscopy. In the case of very large GIST, abdominal CT remains the gold standard is the abdominal CT [11] or MRI in pregnancy, which was perfomed in our case.

All GISTs are potentially malignant, and the risk of recurrence after resection can be assessed according to the size and mitotic index. It is likely that other parameters, such as gastric localization, presence of necrosis and the type of mutation, have prognostic value [12]. GIST metastases are localized in the liver in 2/3 of cases and in the peritoneum in one quarter of cases. Lymph node metastases are rare, not justifying their dissection when the diagnosis is suspected. Lung metastasis are also rare, and their occurrence may justify reviewing the diagnosis. Unfortunately, due to the rarity of the disease there are no recommendations for the management of GIST during pregnancy [6]. Their management differs little from that usually recommended outside of pregnancy and their prognosis is rarely affected by the pregnancy. Outside of the first quarter, there are no great side effects of treatment on the fetus. Preserving fertility is a major challenge for women for whom pregnancy is rarely contraindicated [1].

The only curative treatment of GIST is surgical resection of localized tumors. Metastatic GIST is currently incurable. They are totally resistant to the usual chemotherapy and radiotherapy [6]. The development of targeted therapies specially with inhibitors of tyrosine kinase receptors, such a imatinib mesylate has significantly improved survival of metastatic GIST. Among 4 cases of GIST associated to pregnancy reported in the literature, 3 patients survived at least 9 years after the diagnosis of metastatic GIST [6]. While in the historical series, overall survival of these patients was approximately 18 to 24 months [1]. Imatinib provides a remarkable tumor response in $85 \%$ to $90 \%$ of cases, with progression-free survival of 24 months and overall survival of more than 36 months [13]. Tumor response was significantly correlated with the presence of c-KIT mutations [5]. Adjuvant therapy after complete resection of GIST is probably useful [7]. In addition to surgery, our patient underwent chemotherapy imatinib. Data concerning the use of Imatinib in pregnant women are limited. Studies have shown that imatinib during pregnancy is responsible for spontaneous abortions and congenital anomalies, imatinib should not be used during pregnancy unless clearly necessary. If used during pregnancy, the patient should be apprised of the potential risk to the fetus [1]. The efficacy of imatinib in stromal tumors locally advanced or metastatic is now well established. However, its benefit as adjuvant or neoadjuvant treatment to surgery is not yet fully known [14].

\section{Conclusion}

Gastrointestinal stromal tumors during pregnancy are extremely rare. It is very important to discuss their diagnosis and treatment in multidisciplinary meeting and it is also very important to know their prognostic factors and treatment options. As in our case, multidisciplinary management has better managed the mother and her baby.

\section{Conflict of Interest}

The authors declare that is no conflict of interest.

\section{References}

[1] Cuerva-Gonzalez, M.J., Lacoponi, S., de la Calle-Fernandez, M. and Pozo-Krielinger, J. (2010) Gastrointestinal Stromal Tumor in Pregnancy and Control. Case Report. Ginecologia y Obstetricia de Mexico, 78, 697-702.

[2] Gold, J.S. and Dematteo, R.P. (2006) Combined Surgical and Molecular Therapy: The Gastrointestinal Stromal Tumor Model. Annals of Surgery, 244, 176-184. http://dx.doi.org/10.1097/01.sla.0000218080.94145.cf

[3] Abbas, M., Farouk, Y., Nasr, M.M., Elsebae, M.M., Farag, A., Akl, M.M., et al. (2008) Gastrointestinal Stromal Tu- 
mors (GISTs): Clinical Presentation, Diagnosis, Surgical Treatment and Its Outcome. Journal of the Egyptian Society of Parasitology, 38, 883-894.

[4] Lanteri, R., Aliotta, I., Racalbuto, A. and Licata, A. (2005) Anal GIST in Older Old Patient: A Case Report. Il Giornale di chirurgia, 26, 135-137.

[5] Du, C.Y., Shi, Y.Q., Zhou, Y., Fu, H. and Zhao, G. (2008) The Analysis of Status and Clinical Implication of KIT and PDGFRA Mutations in Gastrointestinal Stromal Tumor (GIST). Journal of Surgical Oncology, 98, 175-178. http://dx.doi.org/10.1002/jso.21104

[6] Emma, T., Igras, A., Susan, J. and Neuhaus, C. (2012) Maternal GIST in Twin Pregnancy: Case Report of a Rare and Complex Management Challenge. Gynecologic Oncology Reports, 2, 133-135. http://dx.doi.org/10.1016/j.gynor.2012.08.001

[7] Chaudhry, U.I. and DeMatteo, R.P. (2009) Management of Resectable Gastrointestinal Stromal Tumor. Hematology/ Oncology Clinics of North America, 23, 79-96. http://dx.doi.org/10.1016/j.hoc.2009.01.001

[8] Neagu, S., Zarnescu, N.O., Costea, R., Stamatoiu, A., Badea, V., Dumitrescu, C., et al. (2003) Gastric Stromal Tumors: Clinical and Histopathological Analysis of Four Cases. Chirurgia, 98, 443-451.

[9] Folgado Alberto, S., Sanchez, P., Oliveira, M., Cuesta, L., Gomes, F., Figueiredo, A., et al. (2008) Gastrointestinal Stromal Tumors-A Retrospective Study of 43 Cases. Revista Espanola de Enfermedades Digestives, 100, 696-700.

[10] Patil, S., Jain, S. and Kaza, R.C. (2011) Chamberlain RS: Giant Gastrointestinal Stromal Tumor Presenting as a Palpable Abdominal Mass: An Unusual Presentation. ISRN Surgery, Article ID: 894829.

[11] Scarpa, M., Bertin, M., Ruffolo, C., Polese, L., D’Amico, D.F. and Angriman, I. (2008) A Systematic Review on the Clinical Diagnosis of Gastrointestinal Stromal Tumors. Journal of Surgical Oncology, 98, 384-392. http://dx.doi.org/10.1002/jso.21120

[12] Emile, J.F., Theou, N., Tabone, S., Cortez, A., Terrier, P., Chaumette, M.T., et al. (2004) Clinicopathologic, Phenotypic, and Genotypic Characteristics of Gastrointestinal Mesenchymal Tumors. Clinical Gastroenterology and Hepatology: The Official Clinical Practice Journal of the American Gastroenterological Association, 2, 597-605.

[13] Heinrich, M.C. (2010) Imatinib Treatment of Metastatic GIST: Don’t Stop (Believing). The Lancet Oncology, 11, 910911. http://dx.doi.org/10.1016/S1470-2045(10)70225-4

[14] Dei Tos, A.P. (2003) GIST: From Diagnosis to Molecular Therapy. Pathologica, 95, 251. 
Scientific Research Publishing (SCIRP) is one of the largest Open Access journal publishers. It is currently publishing more than 200 open access, online, peer-reviewed journals covering a wide range of academic disciplines. SCIRP serves the worldwide academic communities and contributes to the progress and application of science with its publication.

Other selected journals from SCIRP are listed as below. Submit your manuscript to us via either submit@scirp.org or Online Submission Portal.
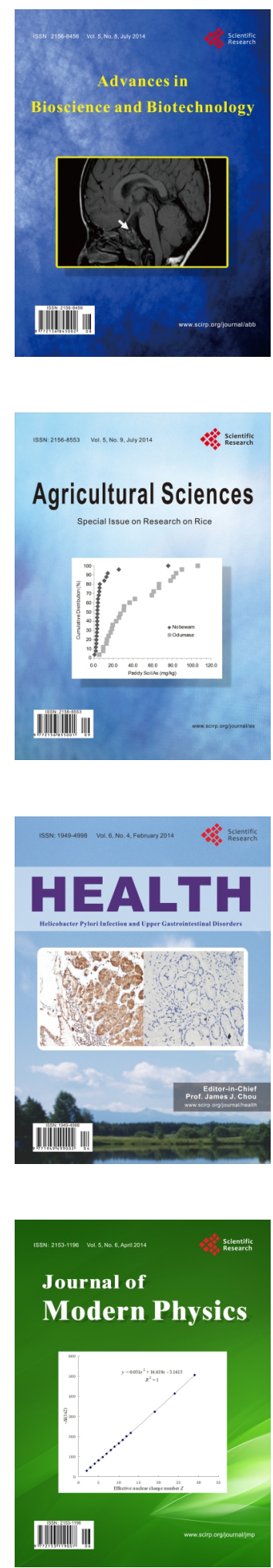
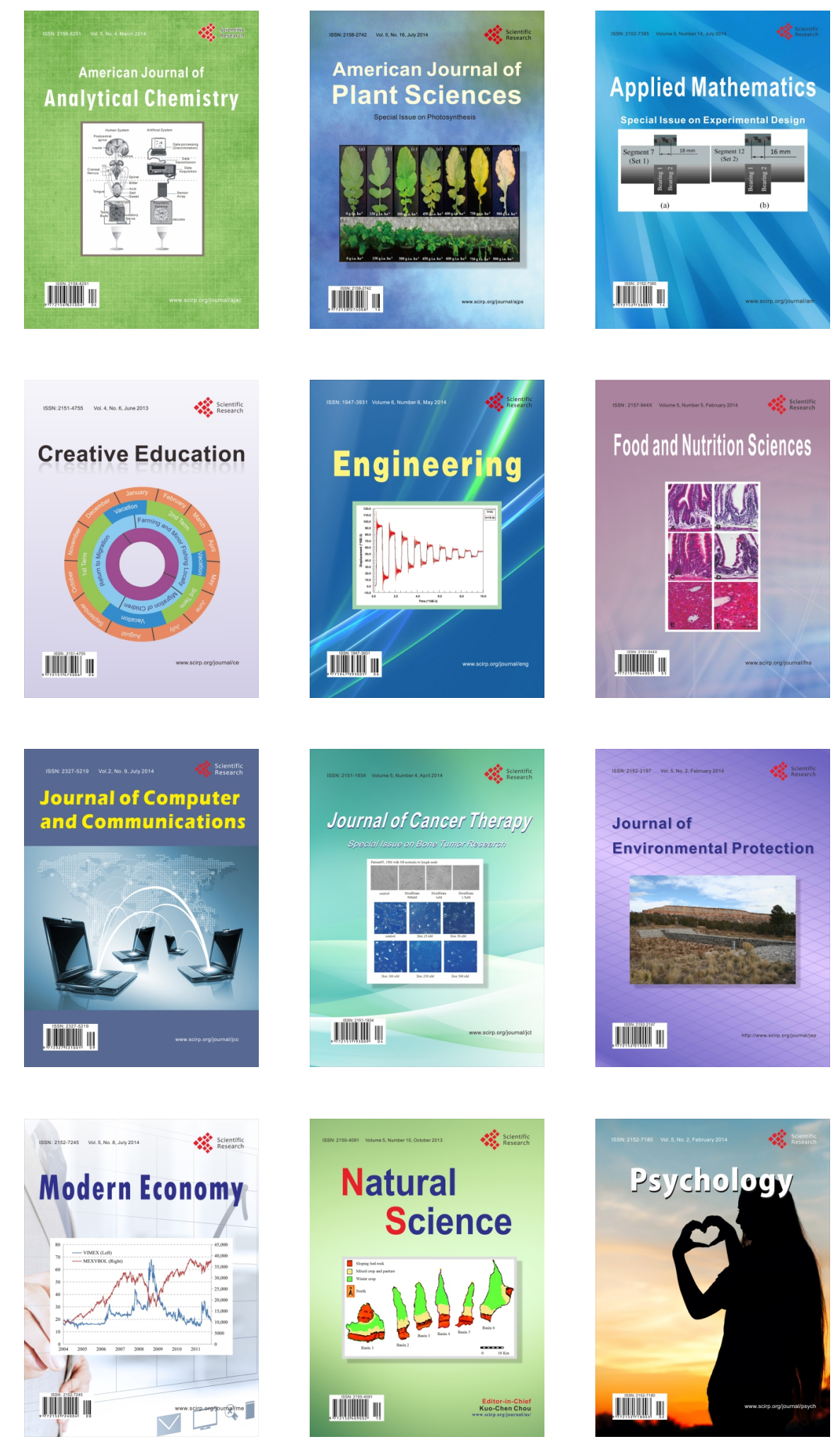